\title{
A Rapid Review of Evaluation Capacity-Building Strategies for Chronic Disease Prevention
}

\author{
Andrea LaMarre \\ Massey University \\ Eric d'Avernas \\ Renison University College \\ Barbara Riley \\ University of Waterloo and Renison University College \\ Amanda Raffoul \\ University of Waterloo \\ Ruchika Jain \\ Independent Researcher
}

\begin{abstract}
There has yet, it seems, to be a review of the literature specifically exploring evaluation capacity building (ECB) for chronic disease prevention (CDP). To guide efforts to build evaluation capacity for $C D P$, a rapid review of the literature was undertaken using systematic methods. A search was conducted of the grey and academic literature to explore ECB strategies in $C D P$, and 14 articles were retained. CDP ECB strategies were similar to general public health ECB efforts (multi-strategy, context-specific, experiential). Articles included a focus on how to maintain ECB over long periods and in light of staff turnover, both of which were described as being prevalent in the CDP context. Evaluating influence at multiple levels (individual, organizational, system) is also important. There is room for more clarity about the "how" of ECB strategies, and about specificity to CDP.
\end{abstract}

Keywords: chronic disease prevention, evaluation capacity, evaluation capacity building

Résumé : À notre connaissance, il n'existe pas encore de recension des écrits traitant du renforcement des capacités en évaluation (RCE) dans le domaine de la prévention des maladies chroniques (PMC). En vue dorienter le RCE dans ce domaine, nous avons entrepris un examen rapide de la littérature, basée sur une approche de recension systématique. Nous avons effectué des recherches dans les littératures grise et scientifique afin d'explorer des stratégies de RCE dans le domaine de la PMC et

Corresponding author: Andrea LaMarre, School of Psychology, Massey University, Level 3

North Shore Library Building, 229 Dairy Flat Highway, Albany, Auckland, New Zealand 0632;

a.lamarre@massey.ac.nz 
avons retenu 14 articles. Les stratégies de RCE en PMC s'apparentaient fortement aux efforts généraux de RCE en santé publique (stratégies multiples, contexte précis, approche expérientielle). Les articles retenus mettaient notamment l'accent sur les façons de maintenir le RCE une fois l'intervention de renforcement terminée et en tenant compte du roulement du personnel, deux facteurs à ne pas négliger dans le contexte de la PMC. Il est aussi important d'évaluer l'influence de ces facteurs à divers niveaux (individuel, organisationnel, systémique). La mise en œuvre des stratégies de RCE et le contexte particulier de la PMC restent encore à clarifier.

Mots clés : prévention des maladies chroniques, capacité en évaluation, renforcement des capacités en évaluation

Evaluation capacity (EC) helps organizations to deliver quality programming; by conducting evaluations and using evaluation results, organizations can make needed improvements to the work that they do (Bourgeois, Simmons, \& Buetti, 2018). EC frameworks suggest that it is important to consider both the capacity to implement ("do") and make use of ("use") evaluation across individual, organization, and pedagogical levels when assessing an organization's EC (Bourgeois \& Cousins, 2013). EC, and the process of building it (termed evaluation capacity building-ECB), has been the focus of studies across subject areas ranging from school counselling (Martin \& Carey, 2012) to governmental organizations (Bourgeois \& Cousins, 2013) and beyond. In this article, we explore ECB in the context of public health, building on broader work to understand effective ECB in public health (e.g., DeCorby-Watson et al., 2018) and looking specifically at ECB in the CDP context.

In general, ECB is conducted intentionally and in context, aims to support both ongoing and prospective work (Stockdill, Baizerman, \& Compton, 2002), and is supported by systemic (e.g., funding, policy), organizational (e.g., resources, leadership), and individual (e.g., knowledge and skills) factors. ECB is best conducted within an overarching evaluation framework (Hotte, Simmons, Beaton, \& the LDCP Workgroup, 2015). Strategies for enhancing ECB include experiential learning, focusing on specific aspects of evaluation, and multi-strategy approaches that target knowledge, interpersonal, behavioural, and programmatic levels (Labin, Duffy, Meyers, Wandersman, \& Lesesne, 2012; Norton, Milat, Edwards, \& Giffin, 2016).

In public health, ECB can have a positive downstream effect on health outcomes (Bourgeois et al., 2018; Bourgeois, Whynot, \& Thériault, 2015; Hotte et al., 2015). A body literature on ECB in public health explores ECB methods across the sector as a whole (e.g., Bergeron et al., 2017; Bourgeois et al., 2018; DeCorbyWatson et al., 2018; Hotte et al., 2015). Like the general ECB literature, this literature highlights the importance of multi-strategy interventions, organizational leadership, and context-specific, realistic approaches to ECB (Bourgeois et al., 2018; DeCorby-Watson et al., 2018). Existing systematic reviews of ECB explore the lack of specificity in the content of ECB interventions, the lack of long-term 
follow-up, and the focus on individual-level, rather than systemic, strategies for ECB (DeCorby-Watson et al., 2018), as well as the often-tacit nature of the theories that underlie ECB activities (Bergeron et al., 2017). This literature reminds us of the importance of accounting for context, enhancing organizational evaluation culture, using methods that integrate experiential and adult learning techniques, and attending to the non-linearity of ECB (Bergeron et al., 2017, DeCorby-Watson et al., 2018). Presently, the ECB literature highlights promising strategies for enhancing EC in organizations but tends not to differentiate between effective strategies across different programmatic areas. The literature review we report on in this article was driven by a desire to better understand which ECB strategies are effective-and why-for chronic disease prevention (CDP).

Within public health, CDP may act as a "catch-all" category; the World Health Organization includes cancer, stroke, heart disease, diabetes, and chronic respiratory diseases (WHO, 2005), as well as HIV (WHO, 2017), within its definition. The 2018 Ontario Public Health Standards (OPHS) use an expansive definition of CDP, encompassing "diseases that are not passed from person to person, are of long duration, and are generally slow in progression" (Ministry of Health and Long-Term Care, 2018, p. 4). Here, mental health and addictions, as well as dementia, "intermediate health states," and hypertension join those diseases characterized as chronic by the WHO. These standards highlight the complex nature of chronic diseases and suggest focusing on risk factors deemed modifiable ("unhealthy eating, physical inactivity, tobacco use, and harmful use of alcohol" [p. 4]) in order to reduce the preponderance of chronic disease. CDP programming often involves multiple actors working together for change over a long period of time and in diverse settings. Hanusaik, O'Loughlin, Kishchuk, Paradis, and Cameron (2010) note that EC for CDP amongst Canadian public health units (PHUs) is lacking, and "because evaluation is key to providing an evidence-base for best practices in CDP programming, [there is a] need for training in evaluation methodology, increased resourcing for evaluation activities, as well as improved funding formulae that recognize and endorse the importance of evaluation" (p. 198).

ECB in CDP is timely: the prevalence of chronic diseases is increasing, and it can be hard to determine whether public health initiatives are working to interrupt disease trajectories. In Ontario, between 2003 and 2013 there were increases in the prevalence of the four leading chronic health conditions: chronic obstructive pulmonary disease (17\% increase), high blood pressure $(42 \%)$, cancer $(44 \%)$, and diabetes (65\%) (Office of the Auditor General of Ontario, 2017a). A significant amount of health-care spending is dedicated to chronic diseases-when combined with injury prevention, chronic disease spending tops $30 \%$ of direct, attributable health-care spending in Ontario (Office of the Auditor General of Ontario, 2017b). The Auditor General recently reported that "the Ministry has not sufficiently supported co-ordination among the PHUs that would help them plan and deliver programs more efficiently" (Office of the Auditor General of Ontario, 2017 b, p. 2), and in Ontario there is "no overarching chronic disease prevention 
strategy" (Office of the Auditor General of Ontario, 2017a, p. 528); PHU capacity to evaluate and carry out CDP programming varies significantly. Developing EC in CDP within PHUs would allow actors in the public health system to work individually and together to foster a nimble and responsive CDP system. To evaluate CDP programs in a high-quality, useful, and sustainable way, those working in the public health system need support and opportunities to build or strengthen relevant capacities.

\section{Research Context}

This literature review was conducted as a part of a two-year grant funded by the Ontario Ministry of Health and Long-Term Care. Our project, CDP-EvaLL, sought to establish systems of learning and support that will embed and sustain ECB for CDP in the Ontario public health sector. CDP-EvaLL aimed to support ECB for public health professionals. This literature review complements a needs assessment by tapping into knowledge about how EC might be built in a way that empowers public health organizations to carry out CDP evaluation. Our focus was specific and action-oriented: we wished to understand which strategies had been used to effectively build EC for CDP. Therefore, we had both project-specific and research contribution aims:

1. to inform the development of ECB for CDP as a part of CDP-EvaLL, alongside a needs assessment;

2. to determine which ECB activities have been effective for CDP programming.

\section{METHODS}

We used systematic search strategies to conduct a rapid review of the academic and grey literature. We followed the National Collaborating Centre for Methods and Tools' Rapid Review Guidebook (Dobbins, 2017), which uses Khangura, Konnyu, Cushman, Grimshaw, and Moher's (2012) focus on the development and delivery of timely evidence in a systematic and rigorous way. We consulted with a librarian at the University of Waterloo to assist with our search. We also selected keywords that aligned with the categories/types of chronic disease outlined in the 2018 OPHS.

We searched several databases selected for their respective foci on Health/ Medicine, Specialized Public Health, and Multidisciplinary academic literature: MEDLINE (via PubMed), Scopus, and CINAHL. We used the search concepts outlined in Table 1. To retrieve as many relevant results as possible, we conducted two searches based around two key concepts: "evaluation capacity" and "capacity building."

After conducting searches in each database with the full search string, we modified terms and fields depending on the database and results retrieved. The 
Table 1. Search terms used in rapid review

\begin{tabular}{|c|c|c|c|c|}
\hline Search 1 & "Evaluation capacity" & $\begin{array}{l}\text { building OR } \\
\text { build OR } \\
\text { development } \\
\text { OR develop }\end{array}$ & $\begin{array}{l}\text { "chronic disease" } \\
\text { OR obesity OR } \\
\text { "cardiovascular } \\
\text { disease" OR } \\
\text { "respiratory } \\
\text { disease" OR } \\
\text { cancer OR } \\
\text { diabetes OR } \\
\text { "metabolic } \\
\text { disease" OR } \\
\text { hypertension } \\
\text { OR dementia OR } \\
\text { "mental illness" } \\
\text { OR addictions }\end{array}$ & $\begin{array}{l}\text { "Public } \\
\text { health" OR } \\
\text { "population } \\
\text { health" }\end{array}$ \\
\hline Search 2 & $\begin{array}{l}\text { "Capacity building" } \\
\text { training OR workshop OR } \\
\text { education[Mesh:noexp] } \\
\text { OR“learning lab" OR } \\
\text { "learning circle" }\end{array}$ & $\begin{array}{l}\text { "program } \\
\text { evaluation" OR } \\
\text { evaluation }\end{array}$ & As above & As above \\
\hline
\end{tabular}

initial results of Search 1 were limited and did not encompass all of the categories of CDP that fall within the OPHS definition; we broadened the search, removing concepts two and three and scanning results manually to retain relevant articles. For Search 2, initial articles were either largely about evaluation of CDP, rather than ECB, or pertained to topics not relevant for our review. The goal was to select reasonably sized yields for the screening process to be completed within the time constraints of the rapid review (a four-week period).

Additionally, we scanned the reference lists of key articles and consulted with experts in the field (members of our project steering committee, $n=9$ ) to obtain additional articles. We conducted a scan of the grey literature for nonacademic works that may be relevant (guided by Godin, Stapleton, Kirkpatrick, Hanning, \& Leatherdale, 2015). We searched the following websites: Public Health Ontario, Canadian Evaluation Society, Ontario Public Health Association, six National Collaborating Centres for Public Health, Ontario Ministry for Health and Long-term Care, and the Custom Search Engines for Canadian Public Health Information and Canadian Public Health Associations. We scanned the first 100 results of each of these searches for relevance. These grey literature searches did not yield retained studies. They did, however, help us to identify academic articles not found using the database search strategies; "grey literature" searches unexpectedly yielded some academic articles, possibly due to the choice of websites to search (e.g., the Canadian Evaluation Society website, which 
Table 2. Inclusion and exclusion criteria

\begin{tabular}{|c|c|}
\hline Inclusion & Exclusion \\
\hline English language & Language other than English \\
\hline Published in/after 1998 & Published before 1998 \\
\hline $\begin{array}{l}\text { Considers ECB for CDP in a Western public } \\
\text { health context }\end{array}$ & Considers ECB in a non-Western setting \\
\hline $\begin{array}{l}\text { Describes methods/tools/strategies used } \\
\text { to build EC }\end{array}$ & $\begin{array}{l}\text { Describes methods/tools/strategies } \\
\text { used to build other skills }\end{array}$ \\
\hline $\begin{array}{l}\text { EC built in the service of enhancing CDP } \\
\text { or the ability to evaluate complex } \\
\text { initiatives }\end{array}$ & General ECB \\
\hline Focuses on ECB methods, tools, strategies & $\begin{array}{c}\text { Focuses on ECB/EC theory (i.e., not tools, } \\
\text { methods, strategies for skill building) }\end{array}$ \\
\hline
\end{tabular}

includes articles from the Canadian Journal of Program Evaluation). Finally, we used the same search terms to search ProQuest Dissertations and Theses.

Using pre-determined inclusion and exclusion criteria (Table 2), two authors (ED, AR) screened titles and abstracts of each academic article retrieved and consulted with a third author (AL) about articles they did not agree upon. Cohen's Kappa was $74.3 \%$ for articles screened at the title/abstract level. In total, 249 academic articles from the searches conducted using database searching strategies were screened at title/abstract level, following removal of duplicates; 29 were retained for full-text screening (see Figure 1). One author (AL) searched for and screened grey literature; of the 324 sources screened, two grey literature sources met inclusion criteria. However, "grey" search strategies also yielded further academic articles, and several sources helped to identify additional academic articles for screening. This included 12 such articles that were retained for full-text screening, and 102 dissertations and theses, from which we retained two. Following full-text screening, 19 academic articles (13 database, six through grey literature) were retained, and two theses/dissertations. Primary reasons for removing articles included the following: not focused on strategies used to build EC, not focused on CDP ECB, and focused on the evaluation itself rather than building capacity for evaluation (Figure 1).

We attempted a critical appraisal of academic articles using a quality appraisal checklist adapted from Critical Appraisal Skills Program (CASP) checklists. However, the structure of the articles did not fit neatly into the categories provided; therefore, quality was assessed on a case-by-case basis. For example, if an article did not include any mention of how the conclusions were reached, we considered it to be of lower quality than an article that described not only the intervention strategy but also its study design. Most articles demonstrated at least medium strength. Three authors extracted data from and discussed the first three articles; the remaining articles were split for extraction: two authors (ED, AR) split 13 academic articles, a third author (AL) extracted data from the grey literature, and one author (ED) extracted data from the theses/dissertations. We put extracted 


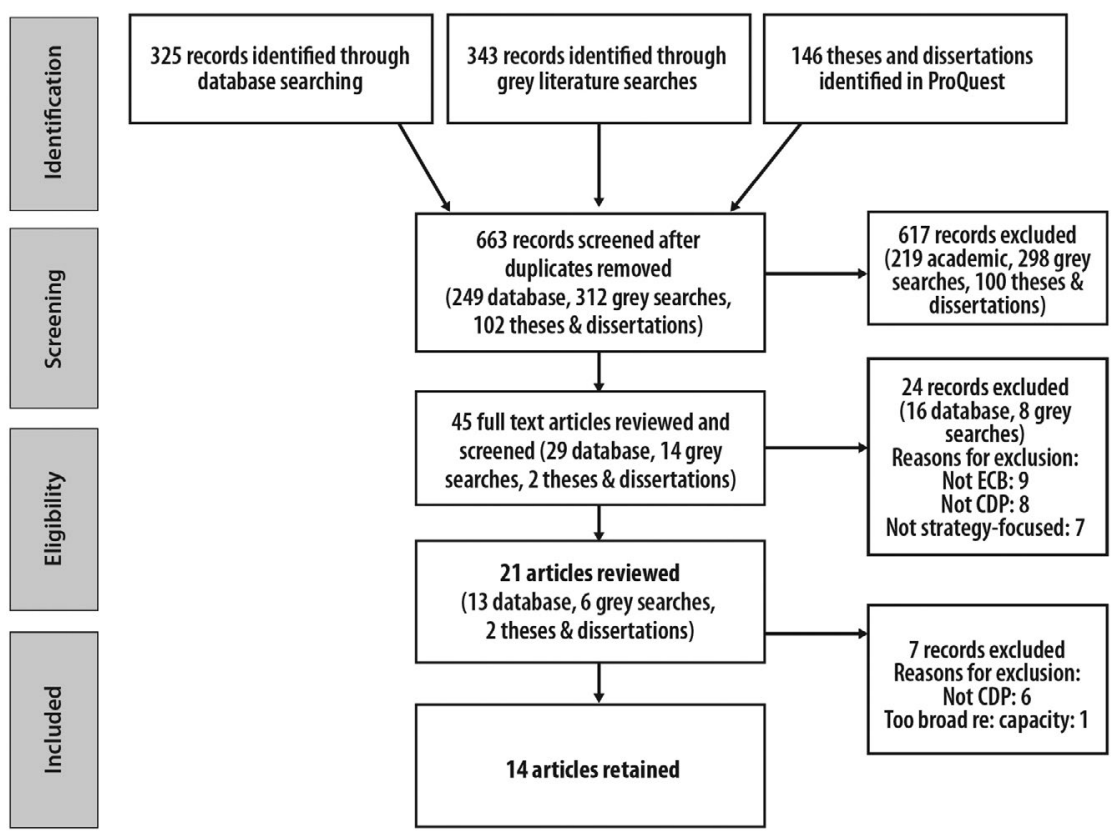

Figure 1. PRISMA diagram

data into Excel spreadsheets, and AL, AR, and ED worked together to synthesize data in turns: ED reviewed the extracted data and created a summary of data; AR and AL then each reviewed this summary and added extra detail and findings. Following review, an additional seven articles were removed because either the focus was not clearly CDP (6) or the article was too broadly focused on organizational capacity, not EC (1).

Data extraction and synthesis were guided by the following questions: What strategies have been used to build EC for CDP in public and population health? How did the authors define CDP in their study? What were the goals of ECB? What was the target of change for ECB? What framework informed the ECB approach? What were the main components of the ECB approaches? How did the authors examine effectiveness? And, finally, what were the results, and how were these affected by contextual factors? These questions were designed to help us to understand the specifics of what those working in CDP saw as key targets for intervention, how they went about making change, and what their outcomes were-in short, deepening our understanding of what ECB looks like for CDP.

For our final analysis, we retained 14 articles about ECB for CDP. These articles focus on ECB strategies for CDP, defined to include "modifiable risk factors" (e.g., nutrition, physical activity, smoking) and conditions included in the 2018 OPHS and WHO definitions (e.g., mental health, cancer, HIV). 


\section{RESULTS}

\section{Domains of CDP}

Of the eight articles that addressed chronic disease directly, one article approached chronic disease generally, one cancer, two mental health, and four HIV. Of the six articles that addressed chronic disease risk/prevention factors, one addressed built environment, two nutrition, and three tobacco. Notably, two of the three articles addressing tobacco were about building EC in the same tobacco control program.

\section{Targets and desired outcomes of ECB}

ECB strategies were targeted at multiple levels, including individual, organizational, and systemic aims. At the individual level, all articles mentioned or alluded to building knowledge and/or skills in conducting evaluation, from planning to implementation, often including the identification, selection, collection, analysis, and use of data. Subsets of evaluation skills targeted include increased awareness and use of evidence-based decision-making (EBDM) skills (Allen et al., 2018), critique of evaluation tools (Dryden, Hyde, Livny, \& Tula, 2010), and the development of partnership-building and community-engagement skills (Kemner, Stachecki, Bildner, \& Brennan, 2015; Kirsh, Krupa, Horgan, Kelly, \& Carr, 2005). Several articles also noted aims related to increasing buy-in to interventions and their evaluation (Dryden et al., 2010; Fourney, Gregson, Sugerman, \& Bellow, 2011; Kirsh et al., 2005). Organizationally, ECB strategies were aimed at increasing access to scientific opportunities (e.g., article access, presentation and publication opportunities) (Allen et al., 2018; Nichols et al., 2018), evaluative thinking and/ or a culture of evaluation within the organization (Compton, Baizerman, Preskill, Rieker, \& Miner, 2001; Compton, MacDonald, Baizerman, Schooley, \& Zhang, 2008; Kirsh et al., 2005; Nu'Man, King, Bhalakia, \& Criss, 2007; Satterlund, Treiber, Kipke, Kwon, \& Cassady, 2013), partnerships with other organizations and/or communities (Compton et al., 2001, 2008; Kirsh et al., 2005), leadership support for evaluation (Nichols et al., 2018; Nu'Man et al., 2007), established feedback systems (Dryden et al., 2010), creation and/or availability of evaluation resources or tools (Dryden et al., 2010; Fourney et al., 2011; Gilliam et al., 2003; Kemner et al., 2015; Kirsh et al., 2005; Lindeman et al., 2018; Naccarella et al., 2007; Nu'Man et al., 2007), and the regular/ongoing provision of external supports for evaluation (Compton et al., 2001, 2008; Treiber, Cassady, Kipke, Kwon, \& Satterlund, 2011). Systemically, articles reported a desire for funding security and sustainability to ensure that ECB efforts are maintained and resourced appropriately (Compton et al., 2008; Nu'Man et al., 2007).

All articles reported that they achieved at least some of their aims through the ECB strategies they used. Notable improvements included self-reported increased skills and/or knowledge (Allen et al., 2018; Compton et al., 2008; Lindeman et al., 2018; Naccarella et al., 2007; Nichols et al., 2018; Nu'Man et al., 2007), creation of or observed improvements to evaluation reports and processes (Allen et al., 2018; Dryden et al., 2010; Fourney et al., 2011; Gilliam et al., 2003; Kemner et al., 
2015; Kirsh et al., 2005; Lindeman et al., 2018; Naccarella et al., 2007; Nu’Man et al., 2007; Satterlund et al., 2013; Treiber et al., 2011), and culture changes and prioritization of evaluation (Allen et al., 2018; Compton et al., 2001, 2008; Dryden et al., 2010; Nu'Man et al., 2007). There were high levels of satisfaction with the ECB strategies amongst those involved, for example, staff who reported feeling that the ECB strategies made the process of evaluation less complicated (Nichols et al., 2018) and strong engagement with technical assistance (Satterlund et al., 2013; Treiber et al., 2011). Satisfaction was sometimes reported in terms of participation rates; here, high participation was related to ECB strategy tailoring to needs (e.g., Dryden et al., 2010; Naccarella et al., 2007) and engagement in ECB strategies led to greater gains, in general (e.g., Lindeman et al., 2018; Nu'Man et al., 2007). Articles varied in the level of clarity they provided for desired outcomes and alignment with achieved changes; some laid out specific aims and reported on outcomes that aligned, whereas others provided a broader description of programming and ECB activities.

\section{ECB strategies}

Strategies noted by authors for building ECB in CDP were similar to findings around ECB strategies noted in reviews of ECB in general (e.g., Norton et al., 2016). All articles used multi-strategy approaches to ECB, most commonly a combination of technical assistance offered in person, via the telephone, or over the Internet, and workshops or training. Eleven articles framed their overall ECB in relationship to technical assistance (Allen et al., 2018; Compton et al., 2001, 2008; Fourney et al., 2011; Gilliam et al., 2003; Kemner et al., 2015; Lindeman et al., 2018; Naccarella et al., 2007; Nu'Man et al., 2007; Satterlund et al., 2013; Treiber et al., 2011). Workshops and training were popular strategies (13 of 14 articles) (Allen et al., 2018; Compton et al., 2001; Compton et al., 2008; Dryden et al., 2010; Fourney et al., 2011; Gilliam et al., 2003; Kemner et al., 2015; Kirsh et al., 2005; Naccarella et al., 2007; Nichols et al., 2018; Nu'Man et al., 2007; Satterlund et al., 2013; Treiber et al., 2011). All articles also noted that they created or provided written and/or online resources as a part of their ECB strategies, to differing degrees (i.e., some provided access, some co-developed, some tailored to context) and of different kinds (e.g., guidelines, toolkits, how-to guides, publications, websites, communication forums, and templates). Sometimes, the development of resources was part of an experiential strategy; this was noted to contribute to the relevance of the resources to the organizational context and/or to the effectiveness of the materials developed due to increased staff buy-in (Dryden et al., 2010; Fourney et al., 2011; Lindeman et al., 2018; Nu'Man et al., 2007).

Eight articles noted using partnership-building or community-engaged strategies to increase EC (Allen et al., 2018; Compton et al., 2001, 2008; Dryden et al., 2010; Fourney et al., 2011; Kirsh et al., 2005; Lindeman et al., 2018; Treiber et al., 2011). Most approaches included active learning strategies or experiential exercises, which sometimes also included an element of mentorship, such that eventually those with less pre-existing evaluation training within the organization 
might be able to conduct the evaluations themselves (e.g., Nichols et al., 2018). Providing ongoing external support was favoured in four articles (Compton et al., 2001, 2008; Satterlund et al., 2013; Treiber et al., 2011). Five articles clearly commented on the need to institutionalize the evaluation process as a part of the ECB plan, for example in the form of guidelines, policies, partnerships, and feedback systems (Compton et al., 2001, 2008; Fourney et al., 2011; Naccarella et al., 2007; Nu'Man et al., 2007). Some articles similarly commented on the need for organizational support for enduring ECB, such as through management and leadership support (Allen et al., 2018; Dryden et al., 2010).

\section{Theories and frameworks informing ECB}

The most commonly used definition for EC was the ability for people within an organization to conduct evaluation within a supportive and well-resourced context-in other words, integrating individual, organizational, and systemic factors; eight of the 14 articles used a variation of this definition (or such a definition could be inferred) (Gilliam et al., 2003; Fourney et al., 2011; Lindeman et al., 2018; Naccarella et al., 2007; Nichols et al., 2018; Nu'Man et al., 2007; Satterlund et al., 2013; Treiber et al., 2011). Three articles described EC in terms of the ability to demonstrate accountability and program improvement (Compton et al., 2001, 2008; Dryden et al., 2010). One article described EC in relation to continuous improvement (Kirsh et al., 2005), one described explicitly participatory EC (Kemner et al., 2015), and one used an EBDM framework in defining EC (Allen et al., 2018). These definitions of EC were sometimes inferred from descriptions of strategies and/or definitions of ECB rather than explicitly defined by authors.

Authors did not always outline their theoretical orientation and how this infused the development of their ECB strategies (see also Bergeron et al., 2017). Five articles described a framework (Fourney et al., 2011; Gilliam et al., 2003; Nu'Man et al., 2007) or taxonomy (Naccarella et al., 2007) or set of guiding principles (Kirsh et al., 2005) that they had designed or adapted to guide their work. Three articles used a utilization-focused framework for ECB either alone (Satterlund et al., 2013; Treiber et al., 2011) or in combination with other theories like collaboration for change and learning organizations (Compton et al., 2001). One article used empowerment evaluation as a framework (Lindeman et al., 2018); the principles of empowerment and/or utilization were also embedded in some of the previously noted frameworks (Fourney et al., 2011; Nu'Man et al., 2007). Two articles focused on participatory evaluations (Dryden et al., 2010; Kemner et al., 2015). One article used a framework for ECB developed by its lead author with colleagues in an earlier ECB initiative, focusing on processes, practices, and roles (Compton et al., 2008).

\section{Contextual factors affecting the success of strategies}

Authors noted that CDP programming often occurs across sites and in diverse contexts (Fourney et al., 2011; Gilliam et al., 2003; Nu'Man et al., 2007). Staff turnover was significant in some CDP contexts, particularly in HIV-prevention 
initiatives and government-level work, leading to losses in capacity with turnover (Compton et al., 2008; Dryden et al., 2010; Gilliam et al., 2003; Lindeman et al., 2018; Naccarella et al., 2007; Satterlund et al., 2013). Staff occupying varied roles are also often involved in CDP programming, making it challenging to appropriately tailor ECB strategies (Naccarella et al., 2007). While this is likely an issue across ECB initiatives, articles commonly reported that within the CDP context there was a need for both individual-level and systemic approaches to ECB to ensure the enduring uptake and continuation of evaluation within organizations (Nichols et al., 2018; Nu'Man et al., 2007).

Authors shared novel approaches to tackling these challenges, for instance the co-production of resources that would be sustained after the initial ECB strategies, with the intention that these materials would reflect the particularized contexts of those working within the organization (Dryden et al., 2010; Fourney et al., 2011; Naccarella et al., 2007; Nu'Man et al., 2007). Selected articles framed technical assistance and external support differently. For some, technical assistance entailed close work with local partners, with technical assistance built into the active phase of ECB (e.g., Nu'Man et al., 2007), whereas for others the focus was on generating sustained relationships (e.g., external consultant/organization-based evaluation expertise: Satterlund et al., 2013; Treiber et al., 2011). Some explored both technical assistance and external support from consultants, framing these as different activities within an overarching ECB strategy (e.g., Gilliam et al., 2003). Students might also be those providing technical assistance, which is a professional development opportunity for them in turn (Compton et al., 2001, 2008).

Collaboration was key to the success of ECB in CDP settings, throughout ECB activities and program development (Compton et al., 2001; Lindeman et al., 2018; Nichols et al., 2018; Nu'Man et al., 2007; Satterlund et al., 2013). Collaboration could include ECB activities and/or program end-users and allows for context-relevant strategy design (Compton et al., 2001; Nu'Man et al., 2007). Another key element of success was the use of multi-strategy approaches to building EC over time (see also Bourgeois et al., 2018; DeCorby-Watson et al., 2018; Norton et al., 2016); ECB “is not simply the provision of training opportunities and workshops that lead to short-term outcomes" (Nu'Man et al., 2007, p. S30).

Authors noted the importance of building sustainability into ECB strategies, for instance acknowledging the role that funders and policymakers can play in maintaining ECB over time (Compton et al., 2001; Kemner et al., 2015; Nu'Man et al., 2007; Satterlund et al., 2013). Articles mentioned that when evaluation outcomes are demonstrable and/or when there is understanding of desired outcomes between stakeholders across the evaluation, program, and funding continuum, there tends to be an increased buy-in to ECB activities (Gilliam et al., 2003; Kirsh et al., 2005; Lindeman et al., 2018; Nu'Man et al., 2007; Satterlund et al., 2013). This is particularly important when funds are in constant flux, for instance in government-funded settings (Compton et al., 2008). Finally, effective ECB is adaptable to ever-changing demands; this is relevant in the CDP context (Gilliam et al., 2003; Satterlund et al., 2013; Treiber et al., 2011), which involves sustaining 
evaluation to fit the longer amount of time between implementation of programming and outcomes.

\section{Limitations of ECB approaches noted in articles}

Articles noted lacking long-term outcome measures and, in some cases, limited generalizability linked to the highly context-dependent nature of their strategies (Allen et al., 2018; Lindeman et al., 2018). Several authors noted that they lacked the time, funding, and resources to conduct long-term follow-up and/or to generate sustainable ECB activities (e.g., Compton et al., 2008; Treiber et al., 2011). Study designs to evaluate the effectiveness of the ECB strategies were often reflective or descriptive (Table 3 ). Authors did not claim that the populations engaged in their ECB efforts and the ECB strategies assessed were representative or generalizable; more often, articles described ECB activities and specified how the described ECB activities met the needs of the particular group(s) with which they were used.

\section{DISCUSSION}

In our review, we found that $\mathrm{ECB}$ in the context of CDP involves using multiple strategies aimed at individual, organizational, and systems levels. These findings echo those of other reviews on ECB in general (Norton et al., 2016) and in public health (DeCorby-Watson et al., 2018) that suggest using multi-strategy interventions for ECB, often including workshops and technical assistance (DeCorbyWatson et al., 2018; Norton et al., 2016). We identified articles that embraced partnership-oriented approaches to ECB (e.g., Dryden et al., 2010; Nichols et al., 2018) and that targeted ongoing ECB throughout a system-particularly in the tobacco-control sector (e.g., Compton et al., 2001; Satterlund et al., 2013; Treiber et al., 2011). Many authors commented on the importance of moving beyond valuing evaluation individually and organizationally toward policy and funder valuing of evaluation to sustain ECB gains (e.g., Nu'Man et al., 2007; Satterlund et al., 2013). This finding extends the work of others who note the critical importance of an organizational culture that values evaluation (e.g., Bourgeois \& Cousins, 2013; Bourgeois et al., 2018) by emphasizing the need for systemic evaluative thinking for sustainability.

This acknowledgment of the need for systemic support helps to explain why despite strong organizational culture and valuing of evaluation, organizations may not always be able to implement ECB. Others have suggested that evaluation must be integrated into the functioning of organizations (e.g., Milstein, Wetterhall, \& CDC Working Group, 2000), but that funding constraints and lack of sustainability might hamper the efforts of those seeking to build EC (Ohmer, 2008); similar challenges exist in research and program systems for CDP across Canada (Johnston, Liddy, Mill, \& Irving, 2012). Despite evidence that those in public health value and wish to engage in ECB (Bourgeois et al., 2018; Hotte et al., 2015), sustainable mechanisms for supporting ongoing ECB in public health have not been established. CDP evaluation presents a challenging puzzle around how 
Table 3. Study design

\begin{tabular}{|c|c|}
\hline Article & Study Design \\
\hline Allen et al. (2018) & $\begin{array}{l}\text { Key informant interviews, quantitative tests through pre/ } \\
\text { post survey }\end{array}$ \\
\hline Compton et al. (2001) & Descriptive and reflective \\
\hline Compton et al. (2008) & Interviews, ECB checklist \\
\hline Dryden et al. (2010) & $\begin{array}{l}\text { Description of intervention with reflections on staff experi- } \\
\text { ences of intervention }\end{array}$ \\
\hline Fourney et al. (2011) & Analysis of final program reports \\
\hline Gilliam et al. (2003) & Descriptive article \\
\hline Kemner et al. (2015) & $\begin{array}{l}\text { Site-specific cases; qualitative, documentation on annual } \\
\text { reports, web-based tracking program }\end{array}$ \\
\hline Kirsh et al. (2005) & $\begin{array}{l}\text { Primarily quantitative data gathering (e.g., reporting on } \\
\text { percentages) from questionnaires, activity observation/ } \\
\text { tracking }\end{array}$ \\
\hline Lindeman et al. (2018) & Survey with retrospective assessment and follow-up \\
\hline Naccarella et al. (2007) & Case study \\
\hline Nichols et al. (2018) & $\begin{array}{l}\text { Rating skills \& confidence (self-report, pre-post), appraisal } \\
\text { of skills \& confidence via evaluation report, quality of } \\
\text { presentation of findings assessed }\end{array}$ \\
\hline Nu'Man et al. (2007) & $\begin{array}{l}\text { Tracking of engagement and skill development; follow-up } \\
\text { contact after 1-6 months }\end{array}$ \\
\hline Satterlund et al. (2013) & $\begin{array}{l}\text { Case study including needs assessment, technical assistance } \\
\text { logs, satisfaction surveys, final evaluation report scores }\end{array}$ \\
\hline Treiber et al. (2011) & $\begin{array}{l}\text { Technical assistance request log, online surveys, changes in } \\
\text { final evaluation report scores }\end{array}$ \\
\hline
\end{tabular}

to support and maintain ongoing ECB, particularly as policies, standards, and people working with CDP portfolios change frequently and often before longterm outcomes have been demonstrated (Gilliam et al., 2003). Within our review, some strategies that authors suggest, including integrating a focus on EC in staff performance reports (Allen et al., 2018), are often only effective as long as staff remain situated at an organization. Staff turnover was noted to be of particular concern in CDP contexts, especially when funding fluctuates and jobs may not be long-term (Compton et al., 2008; Dryden et al., 2010; Naccarella et al., 2007; Satterlund et al., 2013). Systemic approaches to ECB that build in resources and tools for evaluation, as well as an organizational culture that supports evaluation from the leadership levels down, would likely be appropriate in CDP in order to not rely on individual staff members to carry the knowledge and skills needed for evaluation (Dryden et al., 2010). Further, workshops and training may need to be offered in an ongoing way to build out EC within ever-changing organizations (Satterlund et al., 2013).

The results of the review highlight an existing gap around specific strategies designed to enhance ECB for CDP. Many search results were screened out because 
they did not identify specific methods for building EC, and those included did not always include a study design that facilitated generalizability or that clearly identified measurable and replicable changes in EC. Norton et al. (2016, p. 5) suggest that the lack of specificity in terms of ECB impact is at least partly tied to how ECB is often "incorporated within the implementation and evaluation of the program/s for which evaluation capacity is being built." Strategies used to build EC in CDP are similar to those used to build EC in general: for example, the multi-strategy, realistic, organization-wide approaches outlined elsewhere (Bourgeois et al., 2018; DeCorby-Watson et al., 2018; Labin et al., 2012; Norton et al., 2016). This suggests that the general knowledge around ECB can be used to inform ECB in the context of CDP. However, we still lack knowledge of the specific workings of some such strategies, which has implications for the evaluation of ECB in particularized contexts. For our project, this highlighted the importance of evaluating and documenting the steps we took to build EC for CDP in detail to optimize learning from our efforts and make our work replicable.

An ECB approach that uses a combination of internal and external, individu$\mathrm{al}$, organizational, and systemic approaches may be appropriate for CDP because of its often-partnered and collaborative nature, as well as the resource limitations and competing priorities faced by CDP practitioners. Technical assistance can range in intensity and involvement from "on demand" provision of resources and assistance with evaluation plans to one-on-one, extended assistance to regular workshops and support. It may also be present primarily through an active phase of research or on an ongoing basis; target building out organizational EC such that technical assistance is less necessary; or aim to train a set of people who would be able to provide technical assistance continuously. The funding and sustainability of these different approaches may vary, as may the impact on organizational and systemic structures in support of evaluation (or not), which may be more feasible in the short rather than the long term due to staffing and funding complexity, particularly in government-funded work (Compton et al., 2008).

Our review is limited in some ways. First, we attempted critical appraisal, but many articles did not fit the structure for critical appraisal outlined in, for instance, CASP. Several articles held important lessons for CDP ECB but were descriptions of programs rather than complete research designs. Thus, assessments of quality were limited to our assessment of the level of detail provided and reflections on the content of the strategies, and we do not have comparable quality statistics across the data set. Further, we initially targeted "complex" interventions but found that this term was too amorphous and subjective to assess whether articles should be included or excluded. After a thorough review of 21 articles, we eliminated a further seven as they did not directly target CDP. We may have missed relevant strategies that hold promise for ECB CDP in taking this approach. Though not initially anticipated, the strategies that we found for CDP ECB were quite similar to those delineated in the broader ECB literature; this might be framed as a limitation, as we did not gain as much insight into the specificity of 
building EC in the context of CDP, or a positive, as it confirms previous findings and explores the benefits of such strategies for CDP.

Our review provides a glimpse into the types of ECB strategies that might be helpful for CDP. We confirmed our assumption that there is a gap with respect to the specific strategies used for ECB for CDP, and we determined that in the existing literature, similar strategies are used to build EC for CDP as for other public health issues. The importance of multi-strategy, context-specific, dynamic, adaptable, systemic, and sustainable strategies was echoed in the articles included in the review, which has implications for how we think about ECB in CDP. The collaborative, participatory nature of effective strategies outlined in the literature can guide the conceptualization of ECB for CDP programming, which is often multi-site and multi-disciplinary, long-term oriented, and governed by changing systems, thus requiring flexible and creative approaches. In the Ontario context in particular, as noted above, PHUs are not currently supported in the planning and delivery of CDP programs by a comprehensive CDP strategy (Office of the Auditor General of Ontario, 2017b). The results of this review indicate that such support and collaboration are key, including building EC throughout the program planning cycle. To realize the potential benefits of evaluation on health outcomes indicated in the public health evaluation literature (e.g., Bourgeois et al., 2015, 2018; Hotte et al., 2015), ECB efforts must target not only individual capacities to conduct evaluation but also wider organization- and systems-level change in evaluation culture. This support is of particular importance for CDP because of the need for enduring support for long-term programming. In the future, it will be important to consider not only how ECB strategies work in the short term but also how such time- and resource-intensive efforts might gain and sustain traction over the many years between their implementation and the realization of gains in CDP-related outcomes.

\section{ACKNOWLEDGMENTS}

Funded by the Ontario Ministry of Health and Long-term Care, Government of Ontario. The views expressed in the publication are the views of the funding recipient and do not necessarily reflect those of the Province. We would like to thank Jackie Stapleton for her assistance in preparing the search strategy, and members of the CDP-EvaLL team for supporting this review. Authors LaMarre, d'Avernas, Riley, and Jain carried out this work while affiliated with the Propel Centre for Population Health Impact, University of Waterloo.

\section{REFERENCES}

Allen, P., O’Connor, J. C., Best, L. A., Lakshman, M., Jacob, R. R., \& Brownson, R. C. (2018). Management practices to build evidence-based decision-making capacity for chronic disease prevention in Georgia: A case study. Preventing Chronic Disease, 15, E92. https://doi.org/10.5888/pcd15.170482. Medline:30004862 
Bergeron, K., Abdi, S., DeCorby, K., Mensah, G., Rempel, B., \& Manson, H. (2017). Theories, models and frameworks used in capacity building interventions relevant to public health: A systematic review. BMC Public Health, 17(1), 914. https://doi.org/10.1186/ s12889-017-4919-y. Medline:29183296

Bourgeois, I., \& Cousins, J. B. (2013). Understanding dimensions of organizational evaluation capacity. American Journal of Evaluation, 34(3), 299-319. https://doi. org/10.1177/1098214013477235

Bourgeois, I., Simmons, L., \& Buetti, D. (2018). Building evaluation capacity in Ontario's public health units: Promising practices and strategies. Public Health, 159, 89-94. https://doi.org/10.1016/j.puhe.2018.01.031. Medline:29599056

Bourgeois, I., Whynot, J., \& Thériault, E. (2015). Application of an organizational evaluation capacity self-assessment instrument to different organizations: Similarities and lessons learned. Evaluation and Program Planning, 50, 47-55. https://doi.org/10.1016/j. evalprogplan.2015.01.004. Medline:25757074

Compton, D. W., Baizerman, M., Preskill, H., Rieker, P., \& Miner, K. (2001). Developing evaluation capacity while improving evaluation training in public health: The American Cancer Society's Collaborative Evaluation Fellows Project. Evaluation and Program Planning, 24(1), 33-40. https://doi.org/10.1016/s0149-7189(00)00045-8

Compton, D. W., MacDonald, G., Baizerman, M., Schooley, M., \& Zhang, L. (2008). Using Evaluation Capacity Building (ECB) to interpret evaluation strategy and practice in the United States National Tobacco Control Program (NTCP): A preliminary study. Canadian Journal of Program Evaluation, 23(3), 199-224.

DeCorby-Watson, K., Mensah, G., Bergeron, K., Abdi, S., Rempel, B., \& Manson, H. (2018). Effectiveness of capacity building interventions relevant to public health practice: A systematic review. BMC Public Health, 18(1), 684. https://doi.org/10.1186/s12889018-5591-6. Medline:29859075

Dobbins, M. (2017). Rapid review guidebook: Steps for conducting a rapid review. National Collaborating Centre for Methods and Tools. Retrieved from http://www.nccmt.ca/ uploads/media/media/0001/01/a816af720e4d587e13da6bb307df8c907a5dff9a.pdf

Dryden, E., Hyde, J., Livny, A., \& Tula, M. (2010). Phoenix rising: Use of a participatory approach to evaluate a federally funded HIV, hepatitis and substance abuse prevention program. Evaluation and Program Planning, 33(4), 386-393. https://doi.org/10.1016/j. evalprogplan.2010.02.004. Medline:20233628

Fourney, A., Gregson, J., Sugerman, S., \& Bellow, A. (2011). Building evaluation capacity in local programs for multisite nutrition education interventions. Journal of Nutrition Education and Behavior, 43(4 Suppl 2), S130-136. https://doi.org/10.1016/j. jneb.2011.02.005. Medline:21683282

Gilliam, A., Barrington, T., Davis, D., Lacson, R., Uhl, G., \& Phoenix, U. (2003). Building evaluation capacity for HIV prevention programs. Evaluation and Program Planning, 26(2), 133-142. https://doi.org/10.1016/s0149-7189(03)00012-0

Godin, K., Stapleton, J., Kirkpatrick, S. I., Hanning, R. M., \& Leatherdale, S. T. (2015). Applying systematic review search methods to the grey literature: A case study examining guidelines for school-based breakfast programs in Canada. Systematic Reviews, 4(1), 138. https://doi.org/10.1186/s13643-015-0125-0. Medline:26494010 
Hanusaik, N., O’Loughlin, J. L., Kishchuk, N., Paradis, G., \& Cameron, R. (2010). Organizational capacity for chronic disease prevention: A survey of Canadian public health organizations. European Journal of Public Health, 20(2), 195-201. https://doi. org/10.1093/eurpub/ckp140. Medline:19843599

Hotte, N., Simmons, L., Beaton, K., \& the LDCP Workgroup. (2015). Scoping review of evaluation capacity building strategies. Cornwall, ON: Public Health Ontario.

Johnston, S., Liddy, C., Mill, K., \& Irving, H. (2012). Building the evidence base for chronic disease self-management support interventions across Canada. Canadian Journal of Public Health, 103(6), 462-467.

Kemner, A. L., Stachecki, J. R., Bildner, M. E., \& Brennan, L. K. (2015). Increasing community capacity for participatory evaluation of healthy eating and active living strategies through direct observations and environmental audits. Journal of Public Health Management and Practice, 21(Suppl 3), S90-S95. https://doi.org/10.1097/ phh.0000000000000212. Medline:25828230

Khangura, S., Konnyu, K., Cushman, R., Grimshaw, J., \& Moher, D. (2012). Evidence summaries: The evolution of a rapid review approach. Systematic Reviews, 1(1), 10. https:// doi.org/10.1186/2046-4053-1-10. Medline:22587960

Kirsh, B., Krupa, T., Horgan, S., Kelly, D., \& Carr, S. (2005). Making it better: Building evaluation capacity in community mental health. Psychiatric Rehabilitation Journal, 28(3), 234-241. https://doi.org/10.2975/28.2005.234.241. Medline:15690736

Labin, S. N., Duffy, J. L., Meyers, D. C., Wandersman, A., \& Lesesne, C. A. (2012). A research synthesis of the evaluation capacity building literature. American Journal of Evaluation, 33(3), 307-338.

Lindeman, P. T., Bettin, E., Beach, L. B., Adames, C. N., Johnson, A. K., Kern, D., . . Phillips, G. (2018). Evaluation capacity building: Results and reflections across two years of a multisite empowerment evaluation in an HIV prevention context. Evaluation and Program Planning, 71, 83-88. https://doi.org/10.1016/j.evalprogplan.2018.09.001. Medline:30223173

Martin, I., \& Carey, J. C. (2012). Evaluation capacity within state-level school counseling programs: A cross-case analysis. Professional School Counseling, 15(3), 110-122.

Milstein, B., Wetterhall, S. \& CDC Working Group. (2000). A framework featuring steps and standards for program evaluation. Health Promotion Practice, 1(3), 221-228.

Ministry of Health and Long-Term Care [Ontario]. (2018). Chronic disease prevention guideline, 2018. Retrieved from http://health.gov.on.ca/en/pro/programs/publichealth/oph_ standards/docs/protocols_guidelines/Chronic_Disease_Prevention_Guideline_2018.pdf

Naccarella, L., Pirkis, J., Kohn, F., Morley, B., Burgess, P., \& Blashki, G. (2007). Building evaluation capacity: Definitional and practical implications from an Australian case study. Evaluation and Program Planning, 30(3), 231-236. https://doi.org/10.1016/j. evalprogplan.2007.05.001. Medline:17689328

Nichols, N., McFarlane, K., Gibson, P., Millard, F., Packer, A., \& McDonald, M. (2018). Skills, systems and supports: An Aboriginal Community Controlled Health Service (Apunipima) approach to building health promotion evaluation capacity of staff. Health Promotion Journal of Australia, 29(1), 18-22. https://doi.org/10.1002/hpja.18. Medline:29700933 
Norton, S., Milat, A., Edwards, B., \& Giffin, M. (2016). Narrative review of strategies by organizations for building evaluation capacity. Evaluation and Program Planning, 58, 1-19. https://doi.org/10.1016/j.evalprogplan.2016.04.004. Medline:27258905

Nu'Man, J., King, W., Bhalakia, A., \& Criss, S. (2007). A framework for building organizational capacity integrating planning, monitoring, and evaluation. Journal of Public Health Management and Practice, 13, S24-S32. https://doi.org/10.1097/00124784200701001-00006. Medline:17159464

Office of the Auditor General of Ontario. (2017a). Summary: Public health: Chronic disease prevention. Value-for-money audit. Retrieved from http://www.auditor.on.ca/en/ content/news/17_summaries/2017AR\%20summary\%203.10.pdf

Office of the Auditor General of Ontario. (2017b). Chapter 3: Reports on value-for-money audits. 3.10 Public health: Chronic disease prevention. Toronto, ON. Retrieved from http:// www.auditor.on.ca/en/content/annualreports/arreports/en17/2017AR_v1_en_web.pdf

Ohmer, M. L. (2008). Assessing and developing the evidence base of macro practice interventions with a community and neighborhood focus. Journal of Evidence-Based Social Work, 5(3-4), 519-547. https://doi.org/10.1080/15433710802084284. Medline: 19042879

Satterlund, T. D., Treiber, J., Kipke, R., Kwon, N., \& Cassady, D. (2013). Accommodating diverse clients' needs in evaluation capacity building: A case study of the Tobacco Control Evaluation Center. Evaluation and Program Planning, 36(1), 49-55. https:// doi.org/10.1016/j.evalprogplan.2012.05.004. Medline:22784967

Stockdill, S. H., Baizerman, M., \& Compton, D. W. (2002). Toward a definition of the ECB process: A conversation with the ECB literature. New Directions for Evaluation, 2002(93), 7-26. https://doi.org/10.1002/ev.39

Treiber, J., Cassady, D., Kipke, R., Kwon, N., \& Satterlund, T. (2011). Building the evaluation capacity of California's local tobacco control programs. Health Promotion Practice, 12(6 Suppl 2), 118S-124S. https://doi.org/10.1177/1524839911414411. Medline:22068574

World Health Organization (WHO). (2005). Preventing chronic diseases: A vital investment. Geneva, Switzerland: Author. Retrieved from https://www.who.int/chp/chronic_disease_report/contents/en/

World Health Organization (WHO). (2017). Ten years in public health 2007-2017. Geneva, Switzerland: Author. Retrieved from https://www.who.int/publications/10year-review/en/

\section{AUTHOR INFORMATION}

Andrea LaMarre is a lecturer in critical health psychology at Massey University, New Zealand. Prior to taking on this role, she completed a postdoctoral fellowship at the Propel Centre for Population Health Impact, University of Waterloo. She obtained her PhD from the University of Guelph in 2018 and her primary research focus has been on eatingdisorder recovery in social context.

Eric d'Avernas has Master's-level training in French studies, with experience managing a diverse range of projects in both English and French. He has provided evaluation support 
to multi-project initiatives and assisted with a three-workshop series for the Public Health Agency of Canada on systems thinking and public health.

Barbara Riley is Scientific Director, Knowledge Development and Exchange Hub for Child and Youth Mental Health Promotion, Renison University College and Research Associate Professor at the University of Waterloo. Prior to this role, she served as Executive Director of the Propel Centre for Population Health Impact, University of Waterloo. Barb is a health geographer specializing in population health intervention research and evaluation, with a primary focus on multi-site and multi-sector programs in Canada and worldwide.

Amanda Raffoul is a PhD candidate in the School of Public Health and Health Systems at the University of Waterloo. She has expertise in researching public health approaches to dieting, weight management behaviours, prevention of eating- and weight-related disorders, and weight bias. Amanda's work is funded by a Social Sciences and Humanities Research Council (SSHRC) doctoral scholarship.

Ruchika Jain obtained a Master's degree in public health from the London School of Hygiene and Tropical Medicine in 2018. She is interested in evaluating public health interventions aimed at improving the long-term health outcomes of vulnerable persons globally as well as translating research into practice. 